\title{
Constructions de polynômes génériques à groupe de Galois résoluble
}

\author{
par \\ Odile Lecacheux (Paris)
}

1. Introduction. On sait que les seuls sous-groupes résolubles transitifs du groupe symétrique $\mathbf{S}_{5}$ sont isomorphes au groupe de Frobenius $\mathbf{F}_{20}$, au groupe diédral $D_{5}$ et au groupe cyclique $C_{5}$. Nous montrerons comment construire des extensions de degré 5 à groupe de Galois résoluble à l'aide de courbes elliptiques. Dans un premier paragraphe nous utiliserons une courbe elliptique ayant un point de 5-torsion rationnel pour les groupes $D_{5}$ et $C_{5}$. Puis, dans le paragraphe suivant, nous utiliserons une courbe elliptique ayant un sous-groupe rationnel d'ordre 5 pour construire des extensions à groupe de Galois $\mathbf{F}_{20}$. Reprenant alors un résultat de A. Brumer nous obtenons un polynôme générique pour $\mathbf{F}_{20}$.

1.1. Groupe de Frobenius. Rappelons qu'un groupe de Frobenius $G$ de degré premier $p \geq 5$ est un sous-groupe transitif de $S_{p}$ tel que tout élément de $G$ différent de l'identité a au plus un point fixe et qu'il existe un élément ayant un point fixe. Un tel groupe peut s'identifier à un sous-groupe du groupe des transformations affines du corps premier $\mathbb{F}_{p}$, c'est-à-dire du groupe $A_{\mathrm{ff}}\left(\mathbb{F}_{p}\right)=\left\{x \mapsto a x+b: a \in \mathbb{F}_{p}^{*}, b \in \mathbb{F}_{p}\right\}$. Il peut aussi s'identifier au produit semi-direct de $\mathbb{F}_{p}$ par l'unique sous-groupe $H$ d'ordre $l$ divisant $p-1$ de $\mathbb{F}_{p}^{*}$. Si $l=1$ on obtient le groupe cyclique $C_{p}$, si $l=2$ on obtient le groupe diédral $D_{p}$. Dans notre cas si $p=5$, le troisième cas possible correspond à $l=p-1$ et nous noterons $\mathbf{F}_{20}$ ce groupe qui est aussi égal à $A_{\mathrm{ff}}\left(\mathbb{F}_{5}\right)$.

Nous utiliserons dans la suite les représentations de ces groupes à l'aide des permutations $\sigma=(1,4,5,2)$, de carré $\sigma^{2}=(1,5)(2,4)$ et $\tau=(1,2,3,4,5)$. Les deux permutations $\sigma$ et $\tau$ engendrent un groupe isomorphe à $\mathbf{F}_{20}$. Les permutations $\sigma^{2}$ et $\tau$ engendrent un groupe isomorphe à $D_{5}$. $14 \mathrm{~K} 02$.

1991 Mathematics Subject Classification: Primary 12F10, 12F05; Secondary 11G05,

Key words and phrases: polynômes, théorie de Galois, courbes elliptiques. 
1.2. Polynômes génériques. Soit $G$ un groupe fini et $k$ un corps de caractéristique nulle.

DÉFInition 1.1. Un polynôme $P\left(X, n_{1}, \ldots, n_{r}\right) \in k\left[X, n_{1}, \ldots, n_{r}\right]$ est un polynôme générique sur $k$ pour $G$ si

1. comme polynôme en $X$ sur le corps $k\left(n_{1}, \ldots, n_{r}\right)$, un corps de décomposition de $P$ a un groupe de Galois isomorphe à $G$,

2. pour tout corps $K$ contenant $k$ et toute extension $L / K$ galoisienne de groupe $G$, le corps $L$ est le corps de décomposition du polynôme obtenu en spécialisant $P\left(X, n_{1}, \ldots, n_{r}\right)$ en des valeurs $n_{i} \in K$.

1.3. Courbes elliptiques et extensions. On considère une courbe elliptique $E$, définie sur $k$, munie d'une isogénie $k$-rationnelle $\phi$ d'ordre $p$ premier, de noyau engendré par $A$. On notera $E^{\prime}$ la courbe quotient $E /\langle A\rangle$. Soit

$$
y^{2}+a_{1} x y+a_{3} y=x^{3}+a_{2} x^{2}+a_{4} x+a_{6}
$$

une équation de Weierstrass de $E^{\prime}$ et $P^{\prime}=\left(x^{\prime}, y^{\prime}\right)$ un point de $E^{\prime}$ dont l'abscisse $x^{\prime}$ est dans $k$. Soit $P$ tel que $\phi(P)=P^{\prime}$ et soit $k(x)$ l'extension engendrée par l'abscisse $x$ de $P$ dans un modèle de Weierstrass de $E$. Soit $\mathcal{G}$ le groupe de Galois de la clôture galoisienne, sur $k$, du corps de définition de $P$ et $\mathcal{G} \rightarrow G l_{2}\left(\mathbb{F}_{p}\right)$ la représentation de $\mathcal{G}$ définie par $\sigma \mapsto\left(\begin{array}{cc} \pm 1 & 0 \\ b & a\end{array}\right)$ où $P^{\sigma}= \pm P+b A$ et $A^{\sigma}=a A$. De plus, puisque $x$ est une fonction paire sur $E$, le groupe de Galois de la clôture galoisienne de $k(x)$ sur $k$ s'identifie à un sous-groupe du quotient de $G l_{2}\left(\mathbb{F}_{p}\right)$ par le sous-groupe $\pm I_{2}$.

Cette construction et le théorème d'irréductibilité de Hilbert permettent de démontrer, si $p=5$, les résultats suivants :

1. Si $A$ est défini sur $k$, le groupe de Galois de la clôture galoisienne de $k(x)$ est, pour une infinité de $x$, le groupe $D_{5}$.

2. Si $A$ est défini sur une extension cyclique de degré 4 le groupe de Galois de la clôture galoisienne de $k(x)$ est, pour une infinité de $x$, le groupe $\mathbf{F}_{20}$. De même, si $\theta$ est une fonction paire sur $E$ le groupe de Galois de la clôture galoisienne de $k(\theta(P))$ est $\mathbf{F}_{20}$.

2. Groupe cyclique et diédral. Dans ce paragraphe nous expliciterons la construction à l'aide des équations des courbes elliptiques, montrerons qu'on retrouve le polynôme générique donné par A. Brumer et donnerons des exemples.

2.0.1. Notations. Soient $E$ une courbe elliptique définie sur un corps $k$ de caractéristique nulle et $A$ un point de 5 -torsion $k$-rationnel. On notera $A_{i}=i A$ les multiples de $A$. 
Il existe une unique fonction $X$ sur $E$ telle que $X\left(A_{4}\right)=X\left(A_{1}\right)=1$, $X\left(A_{2}\right)=X\left(A_{3}\right)=\infty, X\left(A_{0}\right)=0$. Il en résulte alors que

$$
\operatorname{div}(X)=2 A_{0}-A_{2}-A_{3}, \quad \operatorname{div}(X-1)=A_{1}+A_{4}-A_{2}-A_{3} .
$$

Si $M$ désigne un point générique de $E$, on notera $\Phi$ l'automorphisme du corps des fonctions de $E$ défini par $f(M) \mapsto f(M+A)$, on notera aussi $f_{i}$ la $i$ ème itérée de $f$ par $\Phi$.

2.0.2. Equations de $E$. Il est alors facile de calculer les diviseurs de $X_{i}$ et $X_{i}-1$; plus précisément, on a

$\operatorname{div}\left(X_{i}\right)=2 A_{5-i}-A_{2-i}-A_{3-i}, \quad \operatorname{div}\left(X_{i}-1\right)=A_{1-i}+A_{4-i}-A_{2-i}-A_{3-i}$ pour $0 \leq i \leq 4$.

De l'égalité des diviseurs des fonctions $X_{i}$, on déduit les relations suivantes :

$$
X_{i} X_{i+2}=k^{\prime}\left(1-X_{i+1}\right) .
$$

En considérant les diviseurs des fonctions $X_{i}$, il résulte que le corps des fonctions de $E$ est engendré par $X_{i}$ et $X_{i+1}$. Posons $Y=X_{1}$ et déterminons une relation entre $X$ et $Y$. Cette relation peut être obtenue en considérant la fonction $\prod_{i=0}^{4} X_{i}$. Son diviseur est nul : c'est donc une constante que nous noterons - $t$. Exprimons les différentes fonctions $X_{i}$ à l'aide de $X$ et $Y$. L'automorphisme $\Phi$ étant d'ordre 5 , il en résulte que $k^{\prime}=1$ et on constate que $\Phi(Y)=(1-Y) / X$. On obtient alors une équation de $E$, notée $E_{t}$,

$$
-t X Y=(Y-1)(X-1)(X+Y-1) .
$$

Le changement de variable

$$
X=t / x, \quad Y=1-t x / y
$$

donne le modèle de Weierstrass $E_{t}$ habituellement utilisé (cf. Kubert [4])

$$
y^{2}+(1-t) x y-t y=x^{3}-t x^{2} .
$$

Inversement, si on considère la famille de cubiques $E_{t}$ et si $D=t^{5}\left(t^{2}-\right.$ $11 t-1) \neq 0$ on obtient une courbe elliptique de discriminant $D$, d'invariant

$$
j=-\frac{\left(1-12 t+14 t^{2}+12 t^{3}+t^{4}\right)}{D} .
$$

Dans ce dernier modèle les points de 5 -torsion ont pour coordonnées $A=(t, 0), 2 A=(0,0), 3 A=(0, t), 4 A=\left(t, t^{2}\right)$.

On peut alors expliciter à l'aide de l'automorphisme $\Phi$ l'équation de la courbe $E_{t}^{\prime}$ quotient de $E_{t}$ par le groupe engendré par $A$. Pour cela, remarquons que les fonctions $X-2$ et $X-Y$ ont respectivement 2 et 3 pôles 
simples; posons alors

$$
\begin{aligned}
X^{\prime} & =2 \prod_{i=0}^{4} \Phi^{i}(X-2)=2 \frac{(X-2)\left(X^{2}+2 X t-1\right)\left(2 X^{2}-2 X t-2 X+t\right)}{X(X-1)^{2}}, \\
Y^{\prime} & =4 \prod_{i=0}^{4} \Phi^{i}(X-Y) \\
& =-4 \frac{\left(t X^{2}+(2 X-1)(X-1)^{2}\right)\left((X+1) t-X^{2}(X-1)\right) R(X, Y)}{X^{2}(X-1)^{3}}
\end{aligned}
$$

où $R(X, Y)=X t+(X-1)(X+2(Y-1))$.

Une équation de Weierstrass de $E_{t}^{\prime}$ est alors

$$
\begin{aligned}
Y^{\prime 2}= & X^{\prime 3}+25\left(1+t^{2}\right) X^{\prime 2}+\left(208+76 t+252 t^{2}-76 t^{3}+208 t^{4}\right) X^{\prime} \\
& +4\left(1+t^{2}\right)(-3 t+4)^{2}(4 t+3)^{2} .
\end{aligned}
$$

L'équation aux abscisses liant $X$ et $X^{\prime}$ est

$$
\begin{aligned}
X^{5}+(t-3) X^{4}+\left(1-\frac{1}{4} X^{\prime}-2 t^{2}\right. & \left.-\frac{7}{2} t\right) X^{3}+\left(4 t+3+5 t^{2}+\frac{1}{2} X^{\prime}\right) X^{2} \\
& +\left(-2 t^{2}-2-\frac{1}{4} X^{\prime}-\frac{5}{2} t\right) X+t .
\end{aligned}
$$

2.1. Extension générique à groupe diédral $D_{5}$

ThÉORÈme 2.1 (A. Brumer). Un polynôme générique à groupe de Galois $D_{5}$ sur $k$ est

$$
X^{5}+(s-3) X^{4}+(u-s+3) X^{3}+\left(s^{2}-s-2 u-1\right) X^{2}+u X+s .
$$

Rappelons les grandes lignes de sa démonstration [2] : soient $x_{i}$ les racines d'un polynôme $P(X) \in k[X]$ à groupe de Galois $D_{5}$ représenté à l'aide des permutations $\tau$ et $\sigma$. On pose

$$
X=\frac{\left(x_{4}-x_{2}\right)\left(x_{1}-x_{5}\right)}{\left(x_{4}-x_{1}\right)\left(x_{2}-x_{5}\right)}
$$

birapport de quatre racines. On a alors $k\left(x_{3}\right)=k(X)$ ou bien $X$ est dans $k$. L'action de la permutation $\tau$ d'ordre 5 sur $X$ a même formule que $\Phi$ : plus exactement, $\tau^{2}(X)=(1-\tau(X)) / X$. L'équation aux abscisses $(2.2)$ et le polynôme (2.3) dont les racines sont les $\tau^{i}(X)$ sont identiques en posant $s=t$ et $X^{\prime}=-4 u-8-8 t^{2}-10 t$.

Ceci prouve le corollaire suivant :

Corollaire 2.2. Un polynôme générique $P(X, s, u)$ pour $D_{5}$ sur un corps $k$ est donné par l'équation aux abscisses d'une courbe elliptique $E$ définie sur $k(s)$ munie d'un point de 5-torsion $k(s)$-rationnel. 
2.2. Extensions de degré 5 à groupe cyclique $C_{5}$

ThÉORÈme 2.3. Toute extension galoisienne $L$ de $\mathbb{Q}$ à groupe de Galois cyclique $C_{5}$ est engendrée par les coordonnées d'un point $P$ d'une courbe elliptique $E$ définie sur $\mathbb{Q}$ munie d'un point $A$ de 5 -torsion $\mathbb{Q}$-rationnel dont l'image $P^{\prime}$ dans le quotient $E /\langle A\rangle$ est $\mathbb{Q}$-rationnel. Le point $P$ est ou bien de 25-torsion, ou bien d'ordre infini.

Preuve. Reprenons la construction et les notations du paragraphe précédent. Si l'extension $\mathbb{Q}\left(x_{i}\right)$ est cyclique, $(X, \tau(X)) \in E_{t}\left(\mathbb{Q}\left(x_{3}\right)\right)$ et donc son image dans $E_{t}^{\prime}$ est dans $\mathbb{Q}$.

Si $P$ est d'ordre fini égal à $e$, ses cinq conjugués sont du même ordre donc $e \neq 2,3$. L'extension $\mathbb{Q}\left(x_{i}\right)$ ne contient pas de racines de l'unité $\neq \pm 1$ donc le groupe engendré par $P$ est stable par le groupe de Galois. Le couple $(E, P)$ correspond alors à un point rationnel de la courbe $Y_{0}(5 e)$. La seule possibilité est $e=5$ si le corps de base est $\mathbb{Q}$. Dans ce cas on a $5 P=A$.

On sait alors que ce cas correspond à la famille de corps quintiques suivante (cf. [6]) :

$$
\begin{aligned}
X^{5} & +\left(m^{5}-3\right) X^{4}+\left(-m^{9}-2 m^{8}-3 m^{7}-5 m^{6}-6 m^{5}-2 m^{4}+m^{3}-m^{2}+3\right) X^{3} \\
& +\left(m^{10}+2 m^{9}+4 m^{8}+6 m^{7}+10 m^{6}+9 m^{5}+4 m^{4}-2 m^{3}+2 m^{2}-1\right) X^{2} \\
& -m^{2}\left(m^{7}+2 m^{6}+3 m^{5}+5 m^{4}+5 m^{3}+2 m^{2}-m+1\right) X+m^{5} .
\end{aligned}
$$

Soit avec les notations précédentes :

$$
s=m^{5} \quad \text { et } \quad u=-m^{2}\left(m^{7}+2 m^{6}+3 m^{5}+5 m^{4}+5 m^{3}+2 m^{2}-m+1\right)
$$

et la courbe

$$
y^{2}+\left(1-m^{5}\right) x y-m^{5} y=x^{3}-x^{2} m^{5} .
$$

2.3. Familles de courbes elliptiques de rang $\geq 1$. Il existe des exemples de familles paramétrées d'extensions cycliques de degré 5 ; explicitons les familles de courbes elliptiques dont l'existence est donnée par le théorème 2.3.

2.3.1. Exemple 1. E. Lehmer [7] et R. Schoof-L. Washington [8] ont étudié la famille de corps définie par les polynômes

$$
\begin{aligned}
X^{5}-n^{2} X^{4}+2\left(n^{3}-3 n^{2}+5 n-5\right) X^{3}- & \left(n^{4}-5 n^{3}+11 n^{2}-15 n+5\right) X^{2} \\
& +\left(-n^{3}+4 n^{2}-10 n+10\right) X-1
\end{aligned}
$$

de discriminants

$$
D=\left(-7+10 n-5 n^{2}+n^{3}\right)^{2}\left(n^{4}-5 n^{3}+15 n^{2}-25 n+25\right)^{4} .
$$

Notons $d=\left(n^{4}-5 n^{3}+15 n^{2}-25 n+25\right)$ et $s=-7+10 n-5 n^{2}+n^{3}$, les deux facteurs premiers du discriminant. 
Si $x$ est une racine on vérifie que

$$
z=\frac{x^{2}-n x+n-2}{(n-2) x+1}
$$

est aussi racine, ce qui permet de déterminer l'action de $\tau$ en posant

$$
\tau\left(x_{1}\right)=x_{2}=\frac{x_{1}^{2}-n x_{1}+n-2}{(n-2) x_{1}+1} .
$$

$\mathrm{Si}$

$$
W=\frac{\left(x_{4}-x_{2}\right)\left(x_{5}-x_{1}\right)}{\left(x_{4}-x_{1}\right)\left(x_{5}-x_{2}\right)}
$$

alors $W$ vérifie l'équation

$$
\begin{aligned}
W^{5}+ & \left(n^{3}-10-5 n^{2}+10 n\right) W^{4}+\left(-13 n^{3}+5 n^{4}-5 n+15 n^{2}-n^{5}-10\right) W^{3} \\
& +\left(-160 n+95-8 n^{5}+35 n^{4}+155 n^{2}+n^{6}-91 n^{3}\right) W^{2} \\
& +\left(-20-n^{5}-12 n^{3}+5 n^{4}+10 n^{2}+5 n\right) W-7+10 n-5 n^{2}+n^{3} .
\end{aligned}
$$

Posons

$$
u=-n^{5}+5 n^{4}-12 n^{3}+10 n^{2}+5 n-20 .
$$

On obtient, pour cet exemple, les valeurs de $s$ et $u$ polynôme générique du théorème 2.3 .

Cette famille de corps est obtenue avec la famille de courbes elliptiques

$$
\begin{aligned}
y^{2}-x y(n-2)\left(n^{2}-3 n+4\right)- & \left(n^{3}-5 n^{2}+10 n-7\right) y \\
& =x^{3}-x^{2}\left(n^{3}-5 n^{2}+10 n-7\right)
\end{aligned}
$$

en prenant l'image inverse du point de coordonnées

$$
\begin{aligned}
& x^{\prime}=-8 n^{6}+84 n^{5}-380 n^{4}+950 n^{3}-1350 n^{2}+1000 n-250, \\
& y^{\prime}=4\left(n^{4}-5 n^{3}+15 n^{2}-25 n+25\right)^{2}
\end{aligned}
$$

dans le modèle donné en (2.1).

La courbe (2.4) a un discriminant égal à

$$
\left(n^{2}-5 n+5\right)\left(n^{4}-5 n^{3}+15 n^{2}-25 n+25\right)\left(n^{3}-5 n^{2}+10 n-7\right)^{5} .
$$

Notons le point rationnel $Q$ d'ordre infini sur la courbe (2.4) :

$$
Q=(x=n-1, y=n-2) .
$$

2.3.2. Exemple 2. Dans [9] et [10] G. Smith donne une méthode pour déterminer un polynôme générique pour les extensions cycliques de degré 5 . Après simplification on obtient

$$
P_{C_{5}}(X)=X^{5}+c X^{3}+d X^{2}+e X+f
$$


où

$$
\begin{aligned}
d_{1} & =\left(u^{2}-v^{2}+u v\right)^{2}+5\left(u^{2}+v^{2}+1\right), \\
c & =-50 d_{1}, \quad d=500 d_{1}, \\
e & =625 d_{1}\left(d_{1}-4 u^{2}-4 v^{2}-8\right), \\
f & =-500 d_{1}\left(d_{1}+10 u v\left(u^{2}-v^{2}+u v\right)-10\right) .
\end{aligned}
$$

Le discriminant de $P_{C_{5}}$ est égal à

$$
2^{12} 5^{16} A^{2} B^{2} d_{1}^{4}
$$

où

$$
\begin{aligned}
& A=(7 v-u)\left(u^{2}+u v-v^{2}\right)^{2}+25 v^{2}(u+v), \\
& B=(7 u+v)\left(u^{2}+u v-v^{2}\right)^{2}+25 u^{2}(u-v) .
\end{aligned}
$$

On pose $t=B / A$ et on considère les courbes $E_{t}$ et $E_{t}^{\prime}$.

La courbe $E_{t}$ a un discriminant égal à

$$
125 d_{1}\left(u^{2}+4 u v-v^{2}\right)\left(u^{2}+u v-v^{2}\right)^{2} A^{5} / B^{7}
$$

car

$$
A^{2}+11 A B-B^{2}=125 d_{1}\left(u^{2}+4 u v-v^{2}\right)\left(u^{2}+u v-v^{2}\right)^{2} .
$$

L'extension cyclique est alors le corps de définition du point $P$ de $E_{t}$ dont l'image dans $E_{t}^{\prime}$ (modèle (2.1)) est le point de coordonnées

$$
\begin{aligned}
x^{\prime}= & \left\{2\left(u^{2}-v^{2}+u v\right)^{6}+5\left(u^{2}+v^{2}\right)-10(u+v)(u-3 v)(3 u+v)(u-v)\right. \\
& \left.-5\left(u^{4}+v^{4}\right)-25\left(u^{2}+v^{2}\right)(2 u+v)^{2}(u-2 v)^{2}\right\} / A^{2}, \\
y^{\prime}= & 2^{2} 5^{3} d_{1}^{2}\left(u^{2}+u v-v^{2}\right)^{5} / A^{3} .
\end{aligned}
$$

\section{Polynômes à groupe de Galois $\mathbf{F}_{20}$}

3.1. Isogénies. On considère la courbe elliptique $E_{p}$ d'équation

$$
y^{2}-\frac{d}{4}\left(x^{2}+1\right)=\frac{1}{2} L(x) L^{\prime}(x)
$$

où $L(x)=x^{2}-p x-1, L^{\prime}$ la dérivée de $L$ en $x$ et $d=p^{2}+4$ le discriminant de $L$.

Soient $t$ et $-1 / t$ les deux racines de $L$ et $s$ tel que

$$
s^{2}=\frac{d}{4}\left(t^{2}+1\right)=\frac{d^{3 / 2}}{4} t .
$$

Il est facile de voir que l'extension $\mathbb{Q}(s) / \mathbb{Q}(p)$ est cyclique, de degré 4 et qu'un générateur $\lambda$ du groupe de Galois de $\mathbb{Q}(s) / \mathbb{Q}(p)$ peut être défini par $s \mapsto-s / t$. On vérifie que les points $(t, \pm s)$ et $(-1 / t, \pm s / t)$ sont sur la courbe $E_{p}$ et que la droite passant par les points $(t, s)$ et $(-1 / t,-s / t)$ est tangente en $A=(t, s)$ à la courbe $E_{p}$. Il est alors facile de montrer que le point $A$ engendre un sous-groupe d'ordre 5 stable par le groupe de Galois de $\mathbb{Q}(s) / \mathbb{Q}$. 
On note $E_{p}^{\prime}$ la courbe quotient de $E_{p}$ par le groupe engendré par $A$. est

Si $M=(x, y)$ est un point générique de $E_{p}$ l'abscisse $x_{M+A}$ de $M+A$

$$
x_{M+A}=\frac{y^{2}+(d / 4)\left(t^{2}+1\right)-2 y s}{(x-t)^{2}}-x-t-\frac{1}{4} d+\frac{3}{2} p .
$$

Il en résulte que

$$
\sum_{i=0}^{4} x_{M+i A}=x+2 p+d^{2} \frac{p x+2}{L^{2}}+d \frac{x(p+2)+\left(p^{2}-p+6\right)}{L} .
$$

Posons $r d+5 p / 2=\sum_{i=0}^{4} x_{M+i A}$ et $l=L / d$; alors $l, p, r$ sont liés par la relation

$$
\begin{array}{r}
l^{5}+\left(-r^{2} d+2 p+17 / 4\right) l^{4}+\left(3 r d+p^{2}+13 p / 2+5\right) l^{3}+(r d+11 p / 2-8) l^{2} \\
+(p-6) l-1 .
\end{array}
$$

3.2. Extension générique à groupe de Galois $\mathbf{F}_{20}$

ThÉORÈme 3.1. Soit $k$ un corps de caractéristique nulle. Un polynôme générique $P(X, r, p)$ à groupe de Galois $\mathbf{F}_{20}$ sur $k$ est

$$
\begin{aligned}
X^{5}+\left(-r^{2} d+2 p+17 / 4\right) X^{4}+(3 r d+d+13 p / 2+1) X^{3}+(r d & +11 p / 2-8) X^{2} \\
& +(p-6) X-1
\end{aligned}
$$

où $d=p^{2}+4$.

Preuve. Soit $K$ un corps contenant $k$ et $K^{\prime}=K\left(x_{3}\right)$ une extension de degré 5 de $K$ à groupe de Galois $\mathbf{F}_{20}$.

Nous utiliserons la représentation de $\mathbf{F}_{20}$, à l'aide des permutations, donnée dans l'introduction.

Reprenons les notations précédentes :

$$
X=\frac{\left(x_{4}-x_{2}\right)\left(x_{1}-x_{5}\right)}{\left(x_{4}-x_{1}\right)\left(x_{2}-x_{5}\right)}, \quad Y=X^{\tau} \quad \text { et } \quad-t=\prod_{i=0}^{4} X^{\tau^{i}} .
$$

Il est facile de vérifier que

$$
X^{\sigma}=\frac{\left(x_{2}-x_{4}\right)\left(x_{5}-x_{1}\right)}{\left(x_{2}-x_{1}\right)\left(x_{5}-x_{4}\right)}, \quad Y^{\sigma}=\frac{\left(x_{4}-x_{1}\right)\left(x_{2}-x_{3}\right)}{\left(x_{4}-x_{3}\right)\left(x_{2}-x_{1}\right)} .
$$

Il en résulte que

$$
\begin{gathered}
X^{\sigma}=X /(X-1), \quad Y^{\sigma}=(Y-1) /(X+Y-1), \\
t^{\sigma}=-1 / t, \quad X^{\prime \sigma}=X^{\prime} / t^{2}, \quad Y^{\prime \sigma}=-Y^{\prime} / t^{3} .
\end{gathered}
$$

On pose $T=X . X^{\sigma}, p=t-1 / t, d=p^{2}+4$ et $X^{\prime}+X^{\prime \sigma}=r_{1}\left(p^{2}+4\right)$. 
Il en résulte que $r_{1}$ et $p \in K$ et $T, r_{1}, p$ sont liés par la relation

$$
\begin{array}{r}
T^{5}-(-p+6) T^{4}+\left(-d r_{1} / 2-2 d-11 p / 2+8\right) T^{3}+\left(3 d r_{1} / 2+7 d+13 p / 2+1\right) T^{2} \\
+\left(r_{1}^{2} d / 4+2 r_{1} d+4 d-2 p-17 / 4\right) T+1 .
\end{array}
$$

Si on pose $T=-1 / l, r_{1}=2 r-4$, on retrouve le polynôme construit avec la courbe elliptique $E_{p}$.

Ce polynôme est générique sur $k$ : en effet, il suffit de montrer qu'il existe $x_{3}$ tel que $K^{\prime}=K\left(x_{3}\right)$ et $T \notin K$. Si $T \in K$, alors $T=T^{\tau}$ et $T^{5}=-1$. Si $X=Y$, alors $X$ est racine de $X^{2}+X-1$ et on conclut avec le même argument que pour le cas diédral. Si $X \neq Y$, alors $X=Y /(Y-1)$, ce qui est incompatible avec $T^{5}=-1$.

Remarque 3.1. Le corps de plus petit discriminant ([5]) à groupe de Galois $\mathbf{F}_{20}$, ayant une seule place réelle, est ainsi obtenu pour $p=3, r=$ $-1 / 2$ et le polynôme

$$
X^{5}+7 X^{4}+14 X^{3}+2 X^{2}-3 X-1 .
$$

Il est aussi obtenu avec $p=-3, r=-3 / 2$ et le polynôme

$$
X^{5}-31 X^{4}-64 X^{3}-44 X^{2}-9 X-1 .
$$

Remarque 3.2. Par un choix convenable de $p$ et $r$ on peut obtenir des polynômes à coefficients entiers et, puisque le terme constant est 1 , les extensions de degré 5 sont engendrées par des unités paramétrées. Cette possibilité résulte des propriétés du diviseur de $L$ et du type de mauvaise réduction de $E_{p}$.

Remarque 3.3. L'équation liant les abscisses des points de $E_{p}$ et de $E_{p}^{\prime}$ donne aussi un polynôme générique pour $\mathbf{F}_{20}$; le terme constant est différent de 1 mais les coefficients sont de degré 1 en $x_{2}$.

\section{Références}

[1] A. A. Bruen, C. Jensen and N. Yui, Polynomials with Frobenius groups of prime degree as Galois groups II, J. Number Theory 24 (1986), 305-359.

[2] A. Brumer, preprint.

[3] D. S. Dummit, Solving solvable quintics, Math. Comput. 57 (1991), 387-401.

[4] D. Kubert, Universal bounds on the torsion of elliptic curves, Proc. London Math. Soc. 33 (1976), 193-237.

[5] S. Kwon et J. Martinet, Sur les corps résolubles de degré premier, J. Reine Angew. Math. 375-376 (1987), 12-23.

[6] O. Lecacheux, Unités d'une famille de corps liés à la courbe $X_{1}(25)$, Ann. Inst. Fourier (Grenoble) 40 (1990), 237-253.

[7] E. Lehmer, Connection between Gaussian periods and cyclic units, Math. Comput. 50 (1988), 535-541. 
[8] R. Schoof and L. Washington, Quintic polynomials and real cyclotomic fields with large class numbers, ibid., 543-556.

[9] G. W. Smith, Some polynomials over $\mathbb{Q}(t)$ and their Galois groups, Ph.D. thesis, University of Toledo, 1993.

[10] —, Generic cyclic polynomials of odd order, Comm. Algebra 19 (1991), 3367-3391.

Institut de Mathématiques

Université P. et M. Curie

46-56, 5ème étage, Boîte 247

4 Place Jussieu

75252 Paris Cedex 05, France

E-mail: ol@ccr.jussieu.fr 